\title{
Solar Thermal Plants Integration in Smart Grids
}

\author{
E. F. Camacho* A. J. del Real ${ }^{* *}$ C. Bordons ${ }^{*}$ A. Arce* \\ * Dept. System Engineering and Automation, University of Seville \\ School of Engineering (email: eduardo@esi.us.es, bordons@esi.us.es, \\ aarce@cartuja.us.es) \\ ** IDENER REDD (e-mail: alejandro.delreal@idener.es)
}

\begin{abstract}
Solar energy penetration has been increasingly growing in recent years. Since solar energy is intermittent its integration in existing grids is difficult. This paper deals with the optimal integration of solar power plants in grids. The paper proposes a modification of energy hubs which allows to solve the optimization problem with a mixed integer programming algorithm in a distributed way. An introductory simulation study case is given.
\end{abstract}

\section{INTRODUCTION}

Distributed energy resources, comprising distributed power generators and energy storage units, can play an important role in supporting key policy objectives of combating climate change, increasing the amount of electricity generated from renewable sources, and enhancing energy saving. Distributed generation of electricity, via solar (thermal or photo-voltaics), wind turbines, or combined heat and power plants, has a good chance of penetration in the electricity infrastructure network in the future (Houwing et al. [2007]). Large-scale diffusion of distributed energy resources will have a deep impact on the functioning of the electricity infrastructure: It will bring radical changes to the traditional model of generation and supply as well as to the business model of the energy industry (Houwing et al. [2007]).

In order to cope with this, the electrical power grid is undergoing a major renovation, that will help to meet the power quality and power availability demands of the 21st century. The new power grid, which is also called as the smart grid, aims to integrate the recent technological advancements in the Information and Communication Technology (ICT) field to the power engineering field. The present smart grid implementations focus on smart meter based utility-to-meter and utility-to customer communications. Although these features provide significant improvements on the customer management side, in the following decades, grid management will be one of the major ICTdominant fields. The future of power grids is expected to involve an increasing level of intelligence and integration of new information and communication technologies in every aspect of the electricity system, from demand-side devices to wide-scale distributed generation to a variety of energy markets. However, the most digitized, sophisticated grid in the world will not be really smart if it does not have the capability to put renewable energy online, and make the system more efficient, more reliable and more flexible.

Smart grid technologies are presently undergoing rapid development in an effort to modernize legacy power grids to cope with increasing energy demands of the future
(Amin and Wollenberg [2005]). High speed bi-directional communications networks will provide the framework for real time monitoring and control of transmission, distribution and end-user consumer assets for effective coordination and usage of available energy resources. Furthermore, integration of automation into all levels of power network operations enables smart grids to rapidly self regulate and heal, improve system reliability and security, and more efficiently manage energy delivery and consumption (Garrity [2009]).

With the integration of advanced control algorithms, the smart grid concept will allow to optimize the electrical system by means of the integration of distributed power generation, storage and consumption, absorbing a greater amount of renewable generation while keeping or even improving the current quality standards. Regarding such an integration, this work deals with solar thermal power integration, which has a great interest due to its potential contribution to the energy mix in the following years, and also due to its massive storage capabilities.

The paper is organized as follows: section 2 presents a brief introduction to solar thermal technology; section 3 is related to smart grid management, describing a general modeling and optimization framework in order to fulfil optimal energy grids management; section 4 presents a case study based in the aforementioned optimization framework, showing some interesting aspects related to solar thermal power plants integration in energy networks; finally, section 5 is dedicated to the concluding remarks.

\section{SOLAR THERMAL PLANTS}

The most abundant, sustainable source of energy is the Sun, which provides over 150,000 terawatts of power to the Earth; about half of that energy reaches the Earth surface while the other half gets reflected to outer space by the atmosphere. Only a small fraction of the available solar energy reaching the Earth surface would be enough to satisfy the global expected energy demand. Although most renewable energies derive their energy from the sun, by solar energy we refer to the direct use of solar 


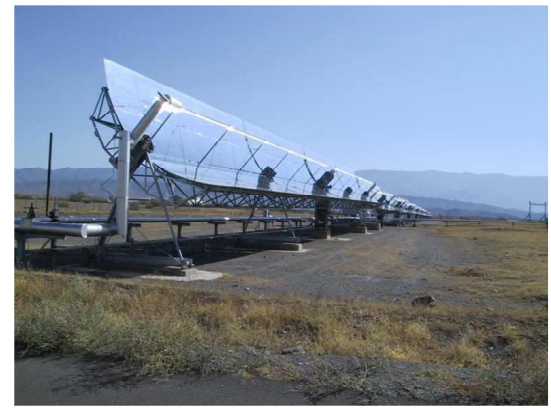

Fig. 1. DISS Parabolic Trough Solar Colector at the PSA

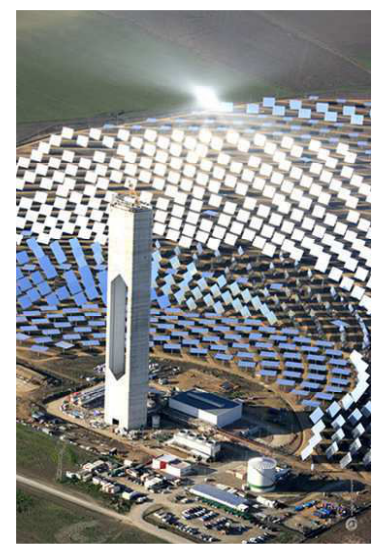

Fig. 2. Abengoa PS-10 Solar Power Tower

radiation. One of the greatest scientific and technological opportunities we are facing is to develop efficient ways to collect, convert, store, and utilize solar energy at affordable costs.

There are two main drawbacks of solar energy systems: a) the resulting energy costs are not yet competitive and b) solar energy is not always available when needed. Considerable research efforts are being devoted to techniques which may help to overcome these drawbacks, control is one of those techniques.

While in other power generating processes, the main source of energy (the fuel) can be manipulated as it is used as the main control variable, in solar energy systems, the main source of power which is solar radiation cannot be manipulated (Camacho et al. [1997]) and furthermore it changes in a seasonal and on a daily base acting as a disturbance when considering it from a control point of view.

Concentrating solar thermal (CST) systems use optical devices (usually mirrors) and sun tracking systems to concentrate a large area of sunlight into a smaller receiving area. The concentrated solar energy is then used as a heat source for a conventional power plant. A wide range of concentrating technologies exist. The main concentrating concepts are: a) parabolic troughs (see Fig. 1), b) solar dishes, c) linear Fresnels, and d) solar power towers (see Fig. 2). The main purpose of the concentrating solar energy is to produce high temperatures and therefore high thermodynamic efficiencies.

Parabolic trough systems are the most used CSP technology. A parabolic trough consists of a linear parabolic mirror that reflects and concentrates the received solar energy onto a tube (receiver) positioned along the focal line. The heat transfer fluid is pumped trough the receiver tube and picks up the heat transferred trough the receiver tube walls. The parabolic mirror follows the Sun by tracking along a single axis.

Regarding the problems that arise due to the the intermittency and unpredictability fluctuations associated to renewable energy resources, massive energy storage should be taken into account. Some technologies related to electricity storage are under intense research: batteries, water pumping, super-capacitors, compressed air, fly wheels, superconducting magnetic energy storages, etc. Among of the most promising of them are those based on hydrogen production and utilization, but none of the aforementioned storage technologies are currently at a commercial stage.

Nonetheless, some modern thermal power plants are incorporating technologies that store energy in a thermal reservoir for a later reuse, which can be employed to balance energy demand between day time and night time. Above some interesting emerging technologies, molten salt has been proposed as a means to retain a high temperature thermal storage for later use in electricity generation. Such thermal reservoirs can store relatively high amounts of energy in periods which can reach 6-8 hours. Storing energy in photovoltaic plants is more costly and difficult. Batteries and Hydrogen fuel cells have been used for this purpose in some experimental installations but there are no photovoltaic plants with a significant storing capabilities.

\section{SMART GRID MANAGEMENT}

\subsection{Grid Mathematical Formulation}

Hybrid energy hubs (see Fig. 3) are defined as interfaces between energy producers, consumers and the transportation infrastructure, introducing a general steadystate modeling and optimization framework for energy systems including multiple energy carriers and describing steady-state power flow couplings between different energy infrastructures and/or network participants.

In particular, hybrid energy hub modeling framework enables integration of an arbitrary number of energy carriers and any technology for transmission, conversion, and storage of energy can be considered. Moreover, such a general formulation ensures high flexibility in terms of modeling detail and accuracy, where more approximate flow models can be used as well as detailed steady-state power flow equations.

As discussed above, the core of the formulation presented herein is composed of three basic features:

- inputs and outputs,

- conversion, and

- storage.

A single converter unit can be seen as shown in Fig. 4. Such an element converts, at time instant $k$, a generic $r$ input flow $u_{i, r}^{L}(k)$ of a generic hub $i$ belonging to a network $\mathcal{N}$ into a generic $p$ output flow $y_{i, p}(k)$, where superscript $L$ is associated to hub variables related to converters. Input- 


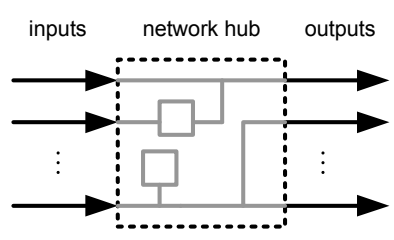

(a)

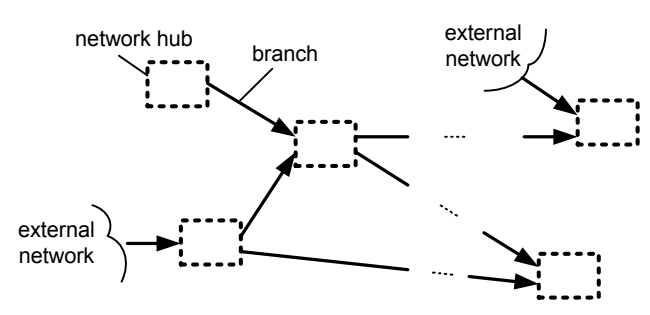

(b)

Fig. 3. (a) network hub sketch and (b) network composed of an interconnection of network hubs

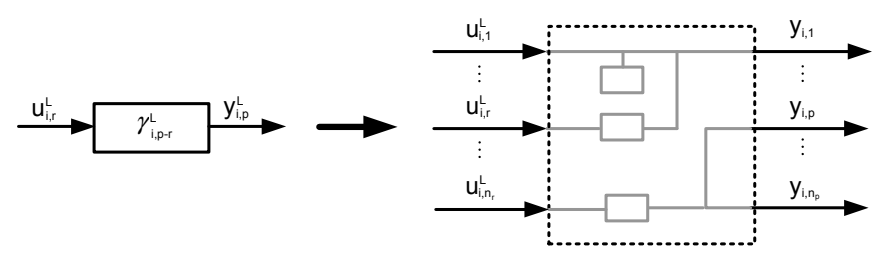

(a)

(b)

Fig. 4. (a) Flow converter with single input and output and (b) converter cluster in a network hub

output conversion is defined through the so-called coupling factors $\gamma_{i, p-r}^{L}$, which correspond to converter's steadystate conversion efficiency between input and output flows:

$$
y_{i, p}(k)=\gamma_{i, p-r}^{L} u_{i, r}^{L}(k) .
$$

A converter set can be expressed through the so-called converter coupling matrix $\Gamma_{i}^{L}$, which describes the mapping of the flows from the input to the output of a hub and is composed of a set of converter coupling factors $\gamma_{i, p-r}^{L}$ :

$$
\underbrace{\left[\begin{array}{c}
y_{i, 1}(k) \\
\vdots \\
y_{i, n_{p}}(k)
\end{array}\right]}_{\mathbf{y}_{i}(k)}=\underbrace{\left[\begin{array}{ccc}
\gamma_{i, 1-1}^{L} & \ldots & \gamma_{i, 1-n_{r}}^{L} \\
\vdots & \ddots & \vdots \\
\gamma_{i, n_{p}-1}^{L} & \cdots & \gamma_{i, n_{p}-n_{r}}^{L}
\end{array}\right]}_{\Gamma_{i}^{L}} \underbrace{\left[\begin{array}{c}
u_{i, 1}^{L}(k) \\
\vdots \\
u_{i, n_{r}}^{L}(k)
\end{array}\right]}_{\mathbf{u}_{i}^{L}} .
$$

For their part, storage devices in hubs are composed of an interface and an internal storage, as shown in Fig. 5. Specifically, the interface can be seen as a flow converter, which modulates a generic $s$ storage interface input flow $u_{i, s}^{E}(k)$ into another generic storage interface output flow $\breve{u}_{i, s}^{E}(k)$. Such an output flow carrier is then stored in an internal ideal storage.

Mathematically, the storage interface is modeled analogously to a converter device, with steady-state input and output flow values being related to each other through the relation:

$$
\breve{u}_{i, s}^{E}=e_{i, s}(k) u_{i, s}^{E}(k),
$$

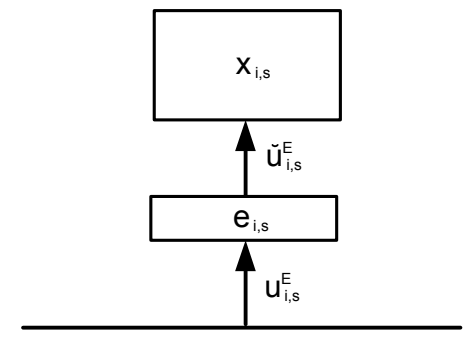

Fig. 5. Storage in network hubs

$e_{i, s}(k)$ being the efficiency of the charge/discharge interface $s$ of hub $i$ belonging to network $\mathcal{N}$, which describes how much of the flow exchanged with the system affects the storage. Such a factor depends on the direction of the exchanged flow or, in other words, the storage can be charged or discharged:

$e_{i, s}(k)=\left\{\begin{array}{lll}e_{i, s}^{+} & \text {if } u_{i, s}^{E}(k) \geq 0 & (\text { charging } / \text { standby }) \\ 1 / e_{i, s}^{-} & \text {else } & \text { (discharging) }\end{array}\right.$

with $e_{i, s}^{+}$and $e_{i, s}^{-}$being the charging and discharging efficiency, respectively. Notice that, for the sake of simplicity, storage performance is assumed to be constant.

Following the formulation, and from a discrete-time point of view, internal storage state $x_{i, s}$ at instant $k+1$ depends on the state at previous instant $k$ and on the total exchanged flow $\breve{u}_{i, s}^{E}(k)$ during the period $\Delta T$ between $k$ and $k+1$, assuming $\breve{u}_{i, s}^{E}(k)$ to remain constant during $\Delta T$ :

$$
\begin{aligned}
x_{i, s}(k+1)=x_{i, s}(k)+ & \int_{k}^{k+1} \breve{u}_{i, s}^{E}(t) d t= \\
& x_{i, s}(k)+\breve{u}_{i, s}^{E}(k) \Delta T .
\end{aligned}
$$

Thus, vector $\mathbf{x}_{i}(k+1)$ containing all the storage states $x_{i, s}(k+1) \in\left\{x_{i, 1}(k+1), \ldots, x_{i, n_{s}}(k+1)\right\}$ depends on the state vector at previous time step $\mathbf{x}_{i}(k)$, on matrix $\Lambda^{E}$ containing all the interface efficiencies $e_{i, s}(k) \in\left\{e_{i, 1}(k)\right.$, $\left.\ldots, e_{i, n_{s}}(k)\right\}$ and on vector $\mathbf{u}_{i}^{E}$ containing all the interface flow inputs $u_{i, s}(k)^{E} \in\left\{u_{i, 1}(k)^{E}, \ldots, u_{i, n_{s}}(k)^{E}\right\}, n_{s}$ being the total number of storage elements in a hub:

$$
\begin{aligned}
& \underbrace{\left[\begin{array}{c}
x_{i, 1}(k+1) \\
\vdots \\
x_{i, n_{s}}(k+1)
\end{array}\right]}_{\mathbf{x}_{i}(k+1)} \underbrace{\left[\begin{array}{c}
x_{i, 1}(k) \\
\vdots \\
x_{i, n_{s}}(k)
\end{array}\right]}_{\mathbf{x}_{i}(k)}+ \\
& \underbrace{\left[\begin{array}{c}
e_{i, 1}(k) \\
\ddots \\
e_{i, n_{s}}(k)
\end{array}\right]}_{\Lambda_{i}^{E}(k)} \underbrace{\left[\begin{array}{c}
u_{i, s}^{E}(k) \\
\vdots \\
u_{i, n_{s}}^{E}(k)
\end{array}\right]}_{\mathbf{u}_{i}^{E}(k)},
\end{aligned}
$$

also taking into account the charging/discharging values of the interface efficiencies, with

$$
\Lambda_{i}^{E}(k)=\left\{\Lambda_{i}^{E+}, \Lambda_{i}^{E-}\right\}=\left[\begin{array}{lll}
\left\{e_{i, 1}^{+}, 1 / e_{i, 1}^{-}\right\} & & \\
& \ddots & \\
& & \left\{e_{i, n_{s}}^{+}, e_{i, n_{s}}^{-}\right\}
\end{array}\right]
$$




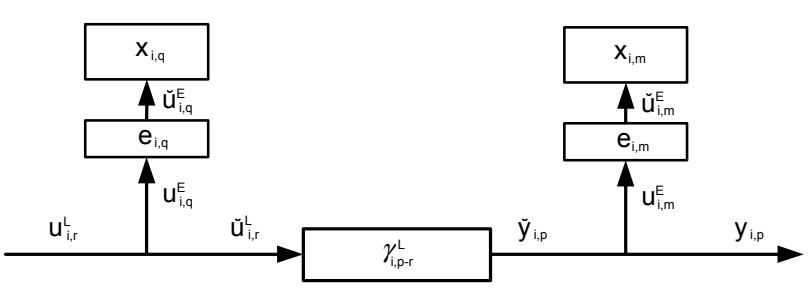

Fig. 6. Multiple storage elements in a network hub

As storage elements may be connected to both hub inputs and outputs (see Fig.6), a mathematical transformation of the corresponding storage flow to either side of the hub has to be considered, in order to obtain an inputoutput description independently where the storage device is connected physically.

As seen in Fig. 6, the flow $\breve{u}_{i, r}^{L}(k)$ entering the converter equals the hub input flow $u_{i, r}^{L}(k)$ minus the left side storage flow $u_{i, q}^{E}(k)$ :

$$
\breve{u}_{i, r}^{L}(k)=u_{i, r}^{L}(k)-u_{i, q}^{E}(k) .
$$

Analogously, the output converter side can be described as:

$$
\breve{y}_{i, p}(k)=y_{i, p}(k)+u_{i, m}^{E}(k)
$$

where $\breve{y}_{i, p}^{L}(k)$ is the flow leaving the converter, $y_{i, p}$ is the hub output flow and $u_{i, m}^{E}(k)$ is the right side storage flow. Expressing flow transformations as in (1) yields to:

$$
\breve{y}_{i, p}(k)=\gamma_{i, p-r}^{L} \breve{u}_{i, r}^{L}(k) .
$$

Substituting (8) and (9) in (10), both converter sides storage flows can be explicitly included in the hub inputoutput description:

$$
\begin{aligned}
y_{i, p}(k)= & \gamma_{i, p-r}^{L}\left(u_{i, r}^{L}(k)-u_{i, q}^{E}(k)\right)-u_{i, m}^{E}(k)= \\
& \gamma_{i, p-r}^{L} u_{i, r}^{L}(k)+\gamma_{i, p-r}^{E} u_{i, r}^{E}(k),
\end{aligned}
$$

where $\gamma_{i, p-r}^{E}$ are the coupling factors related to storage variables $u_{i, r}^{E}(k)$. Following the discussion, vector $\mathbf{y}_{i}(k)$ containing all hub outputs $y_{i, p}(k) \in\left\{y_{i, 1}(k), \ldots, y_{i, n_{p}}(k)\right\}$ can be extended in order to include storage elements through the so-called storage coupling matrix $\Gamma_{i}^{E}$. Such a matrix describes how changes of the storage flows affect the converter output flows, resulting the following equation:

$$
\begin{gathered}
\underbrace{\left[\begin{array}{c}
y_{i, 1} \\
\vdots \\
y_{i, n_{p}}
\end{array}\right]}_{\mathbf{y}_{i}}=\underbrace{\left[\begin{array}{ccc}
\gamma_{i, 1-1}^{L} & \cdots & \gamma_{i, 1-n_{r}}^{L} \\
\vdots & \ddots & \vdots \\
\gamma_{i, n_{p}-1}^{L} & \cdots & \gamma_{n_{p}-n_{r}}^{L}
\end{array}\right]}_{\Gamma_{i}^{L}} \underbrace{\left[\begin{array}{c}
u_{i, 1}^{L}(k) \\
\vdots \\
u_{i, n_{r}}^{L}
\end{array}\right]}_{\mathbf{u}_{i}^{L}}+ \\
\underbrace{\left[\begin{array}{ccc}
\gamma_{i, 1-1}^{E} & \cdots & \gamma_{i, 1-n_{s}}^{E} \\
\vdots & \ddots & \vdots \\
\gamma_{i, n_{p}-1}^{E} & \cdots & \gamma_{n_{p}-n_{s}}^{E}
\end{array}\right]}_{\Gamma_{i}^{E}} \underbrace{\left[\begin{array}{c}
u_{i, 1}^{E}(k) \\
\vdots \\
u_{i, n_{s}}^{E}
\end{array}\right]}_{\mathbf{u}_{i}^{E}} .
\end{gathered}
$$

Summarizing equations (6) and (12) yields to a complete state-state representation of a generic hub $i \in \mathcal{N}$ :

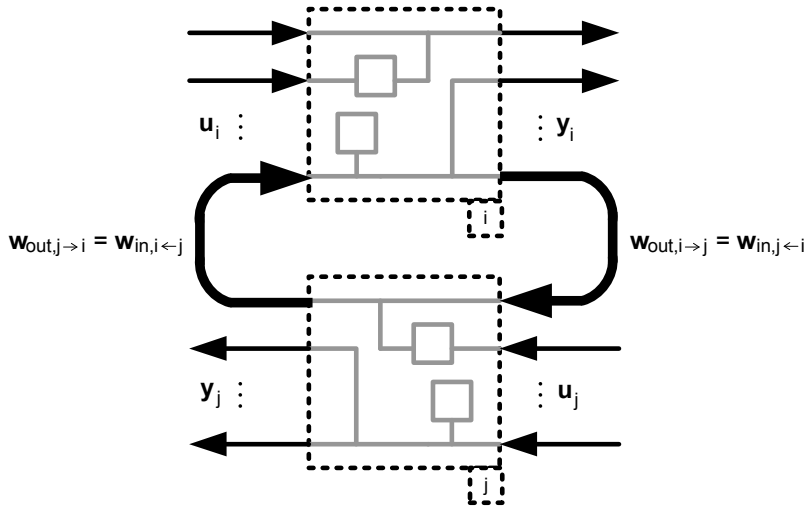

Fig. 7. Interconnections between hubs i and j

$$
\begin{aligned}
\mathbf{x}_{i}(k+1) & =\mathbf{x}_{i}(k)+\Lambda^{E} \mathbf{u}_{i}^{E}(k) \\
\mathbf{y}_{i}(k) & =\Gamma_{i}^{L} \cdot \mathbf{u}_{i}^{L}(k)+\Gamma_{i}^{E} \cdot \mathbf{u}_{i}^{E}(k),
\end{aligned}
$$

which can be condensed by defining the complete converter interface efficiency matrix $\Lambda_{\mathbf{i}}$, the complete coupling matrix $\Gamma_{\mathbf{i}}$ and the complete hub input vector $\mathbf{u}_{\mathbf{i}}(k)$ as follows:

$$
\begin{aligned}
\Lambda_{i}(k) & =\left[\mathbf{0} \mid \Lambda_{i}^{E}(k)\right] \\
\Gamma_{i} & =\left[\Gamma_{i}^{L} \mid \Gamma_{i}^{E}\right] \\
\mathbf{u}_{i} & =\left[\mathbf{u}_{i}^{L}(k)^{T} \mid \mathbf{u}_{i}^{E}(k)^{T}\right]^{T},
\end{aligned}
$$

which results in the next condensed state space representation:

$$
\begin{aligned}
\mathbf{x}_{i}(k+1) & =\mathbf{x}_{i}(k)+\Lambda_{i}(k) \mathbf{u}_{i}(k) \\
\mathbf{y}_{i}(k) & =\Gamma_{i} \mathbf{u}_{i}(k) .
\end{aligned}
$$

From this formulation it is clear that a generic network hub $i$ can be fully described by the set of matrices $\mathcal{H}_{i}=\left\{\Lambda_{i}(k), \Gamma_{i}\right\}$, which is the main advantage of the formulation proposed herein, as it offers a simple method to model arbitrarily complex systems.

Regarding a complete energy grid, let's consider a generic network composed of a set of $\mathcal{N}=\left\{1, \ldots, n_{\mathcal{N}}\right\}$ interconnected hubs (see Fig. 7), $n_{\mathcal{N}}$ being the total number of hubs composing the network. The dynamics of subnetwork $i \in \mathcal{N}$ is defined by the following nonlinear time-invariant discrete-time state space model:

$$
\begin{aligned}
\mathbf{x}_{i}(k+1) & =\mathbf{x}_{i}(k)+\Lambda_{i} \mathbf{u}_{i}(k) \\
\mathbf{y}_{i}(k) & =\Gamma_{i} \mathbf{u}_{i}(k)+\Pi_{i n, i} \mathbf{w}_{i n, i}(k),
\end{aligned}
$$

Considering the interconnections among hubs, generic interconnecting variables between hub $i$ and its neighbor $j$ can be defined as seen in Fig. 7, where $\mathbf{w}_{i n, i \leftarrow j}(k)$ and $\mathbf{w}_{\text {out }, i \rightarrow j}(k)$ are, respectively, generic interconnecting input and output vectors of hub $i$ related to its neighboring hub $j$, with:

$$
\mathbf{w}_{\text {out }, i \rightarrow j}(k)=\Pi_{\text {out }, i \rightarrow j} \mathbf{y}_{i}(k),
$$

where $\Pi_{o u t, i \rightarrow j}$ is the output interconnecting matrix referred to the coupling of hub $i$ with respect to its neighbor $j$. 


\subsection{Grid Distributed Management}

A Model Predictive Control (MPC), (Camacho and Bordons [2004])Due to the complexity of future energy networks, with an increasing number of consumers and producers, a distributed control effort is taken into account in this work. Specifically, a Lagrange-Based Distributed Model Predictive Control (Lag-MPC) formulation (Negenborn [2007]) has been chosen as the control framework utilized in order to solve the network problem. The objective of such a problem is to minimize the total cost of supplying energy to satisfy the demand. To that end, each energy producer has assigned a cost per power unit produced while, at the same time, each consumer has assigned an energy demand.

The next algorithm implements the Lag-MPC discussed herein, which would have to be done, at each time step $k$, by each local agent $i \in \mathcal{N}$ in a parallel computation scheme:

(1) Make a measurement of current state $\widehat{\mathbf{x}}_{i}(k)$

(2) Compute the optimal control sequence $\widetilde{\mathbf{u}}_{i}^{*}(k)$. To do so, perform the following steps:

(a) Parameter initialization:

$$
p=1 ; e_{i} \gg 1 ; \lambda_{i n, i \rightarrow j}^{1}(k)=0 ; \lambda_{\text {out }, i \rightarrow j}^{1}(k)=0
$$

(b) Solve the following optimization problem:

$$
\begin{aligned}
& \min _{\begin{array}{r}
\widetilde{\mathbf{x}}_{i}(k+1), \widetilde{\mathbf{u}}_{i}(k), \widetilde{\mathbf{y}}_{i}(k) \\
\widetilde{\mathbf{w}}_{\text {in }, i}(k), \widetilde{\mathbf{w}}_{\text {out }, i}(k)
\end{array}} \phi_{\text {local }, i}\left(\widetilde{\mathbf{x}}_{i}(k+1), \widetilde{\mathbf{u}}_{i}(k), \widetilde{\mathbf{y}}_{i}(k)\right) \\
& \quad+\sum_{j \in \mathcal{N}_{i}} \phi_{\text {inter }, i}\left(\widetilde{\mathbf{w}}_{\text {in }, i}(k), \widetilde{\mathbf{w}}_{\text {out }, i}(k)\right),
\end{aligned}
$$

subject to subnetwork dynamics, constraints and initial condition.

(c) Send $\widetilde{\mathbf{w}}_{i n, i \rightarrow j}^{p}(k)$ and $\widetilde{\mathbf{w}}_{\text {out }, i \rightarrow j}^{p}(k)$ to neighboring agents $j \in \mathcal{N}_{i}$ and collect $\widetilde{\mathbf{w}}_{i n, j \rightarrow i}^{p}(k)$ and $\widetilde{\mathbf{w}}_{\text {out }, j \rightarrow i}^{p}(k)$ from them. In other words, you (an agent $i$ ) tells to your neighbors $j \in \mathcal{N}_{i}$ what you would like to do $\widetilde{\mathbf{w}}_{\text {out }, i \rightarrow j}^{p}(k)$ and what you would like them to do $\widetilde{\mathbf{w}}_{i n, i \rightarrow j}^{p}(k)$. At the same time, you receive what your neighbors want to do $\widetilde{\mathbf{w}}_{\text {out }, j \rightarrow i}^{p}(k)$ and also what your neighbors want you to do $\widetilde{\mathbf{w}}_{i n, j \rightarrow i}^{p}(k)$.

(d) Upgrade the Lagrange multipliers

$$
\begin{aligned}
\widetilde{\lambda}_{i n, i \rightarrow j}^{p+1}(k)= & \widetilde{\lambda}_{i n, i \rightarrow j}^{p}(k)+ \\
& \gamma_{c}\left(\widetilde{\mathbf{w}}_{i n, i \rightarrow j}^{p}(k)-\widetilde{\mathbf{w}}_{\text {out }, j \rightarrow i}^{p}(k)\right) \\
\widetilde{\lambda}_{\text {out }, i \rightarrow j}^{p+1}(k)= & \widetilde{\lambda}_{\text {out }, i \rightarrow j}^{p}(k)+ \\
& \gamma_{c}\left(\widetilde{\mathbf{w}}_{\text {out }, i \rightarrow j}^{p}(k)-\widetilde{\mathbf{w}}_{\text {in }, j \rightarrow i}^{p}(k)\right) .
\end{aligned}
$$

(e) Evaluate the stopping conditions:

$$
\begin{aligned}
& \text { - } p>\bar{p} \\
& \text { - } e_{i}=\left\|\widetilde{\lambda}_{i n, i \rightarrow j}^{p+1}(k)-\widetilde{\lambda}_{i n, i \rightarrow j}^{p}(k)\right\| \leq \bar{e},
\end{aligned}
$$

where $\bar{p}$ is the maximum number of iterations allowed and $\bar{e}$ is the maximum error allowed. If one of the stopping conditions is true, go to step (3). If not, move on to the next iteration $p \leftarrow p+1$ and return to step (b).

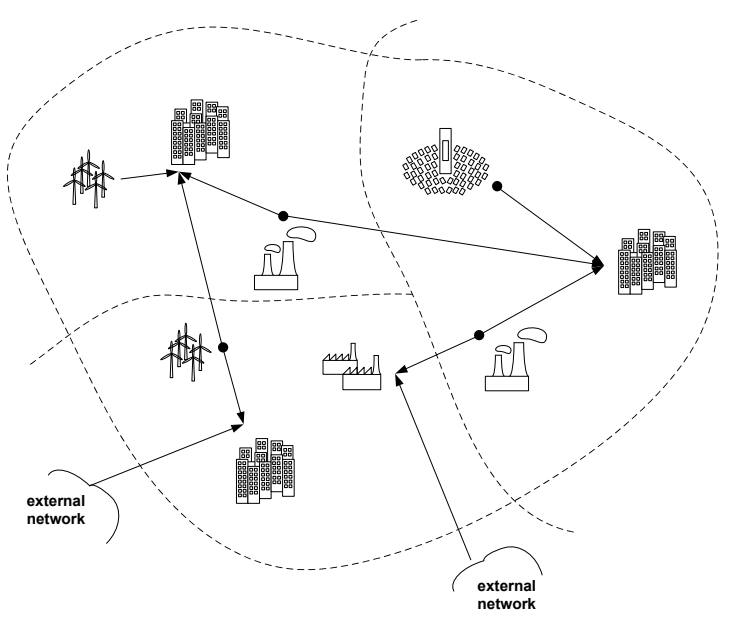

Fig. 8. Energy network case study

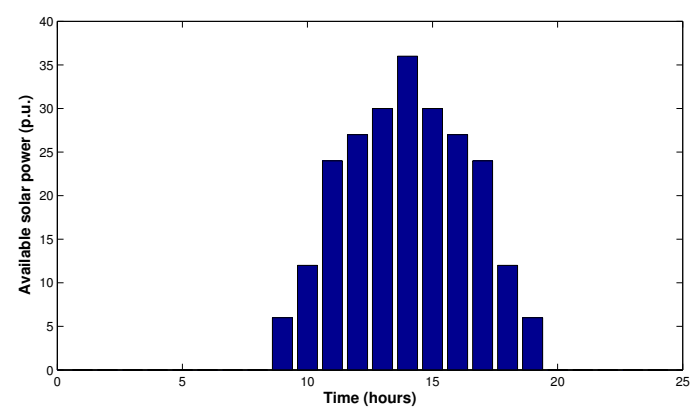

Fig. 9. Available solar power (p.u.)

(3) Implement the optimal control action $\mathbf{u}_{i}^{*}(k)$

(4) Start a new control cycle $k \leftarrow k+1$ and go to step (1)

\section{CASE STUDY}

In this section, a network case study is studied in order to show an example of a solar thermal plant integration in a smart grid. Specifically, the energy network shown in Fig. 8 was simulated. Such a network is composed of three interconnected city centers, an industrial center, two wind farms, two thermal fuel oil plants and a solar thermal plant.

The aforementioned network case study was simulated in MATLAB, also utilizing CPLEX solver. Such a network was firstly divided into three sectors, thus considering three interconnected network hubs. Each hub was assigned to a Lag-MPC control agent, and the distributed control algorithm described in the preceding section was performed. A 24-step simulation time was considered, thus dividing a complete day into 24 hours. Regarding the thermal solar plant and due to the solar irradiation data taken herein into account, total solar power shown in Fig. 9 corresponding to a sunny day and expressed in power units (p.u.) was available.

As can be seen in Fig. 8, the thermal solar plant is directly connected to a city center, which is also feed by the two fuel oil power plants. In order to simulate the network, each energy producer offers a certain energy to the consumers each time step. At the same time, each 


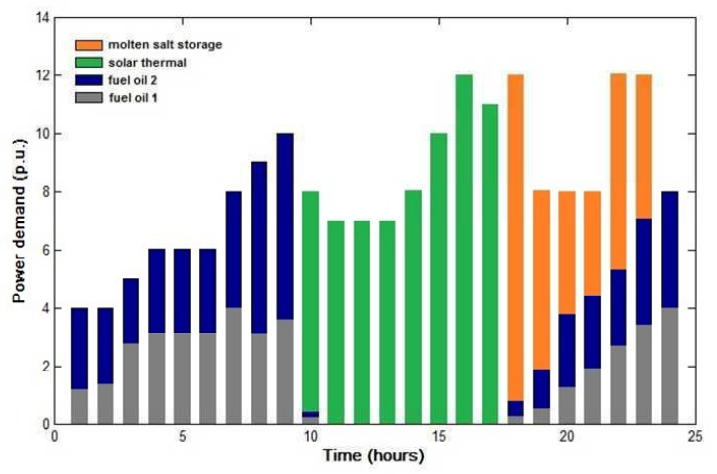

Fig. 10. Power demand energy mix
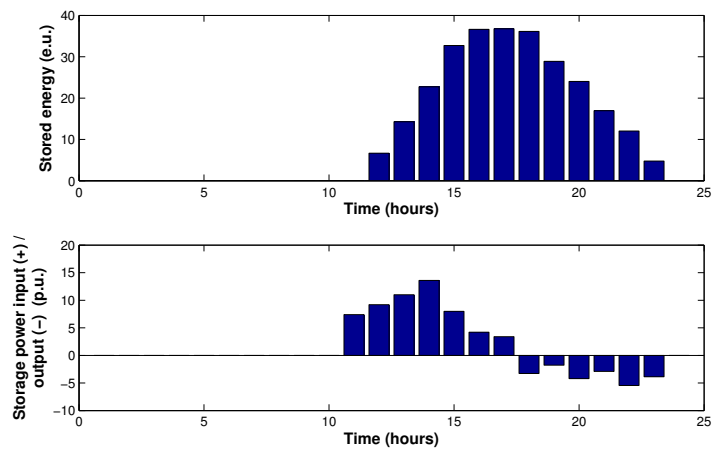

Fig. 11. Stored energy (e.u.) (upper graph) and storage power input and output (p.u.) (lower graph)

consumer minimizes their total demanded energy cost by selecting the best offers each time step.

In this example, investment and operational costs of the thermal solar plant are supposed to be supported by feed in tariffs and only fuel costs are considered. Since the thermal power plant "raw material" is solar irradiation, such a plant can offer its energy production at a cost of 0 monetary units (m.u.) per power unit (p.u.) produced. In turn, the fuel oil power plants offer, respectively, power at a cost of 2.5 and 3 m.u. per p.u. As a result, all solar power offered is bought by the city center.

In order to show the impact of energy storage in solar thermal plants, a energy storage device (such as molten salt) was firstly taken into account. Fig. 10 shows the resulting power demand mix of the city center placed next to the solar thermal plant. As can be seen, first nine steps are dominated by fuel oil power, as no power from sun is available. Contrarily, sun power is mainly demanded the following eight hours. At the same time, as can be seen in Fig. 11, sun power excess is stored in the thermal solar plant storage device, resulting in an increase in the energy stored (Fig. 11 upper graph). At time step 20, no more solar irradiation is available. However, as there is a sensible energy amount stored in the molten salt device, the solar thermal power plant is able to continue injecting power to the grid. As a result, power from the storage device is also included in the energy mix corresponding to the last part of the simulation.

Finally, Fig. 12 shows a comparison between the described scenario with a solar thermal plant including storage

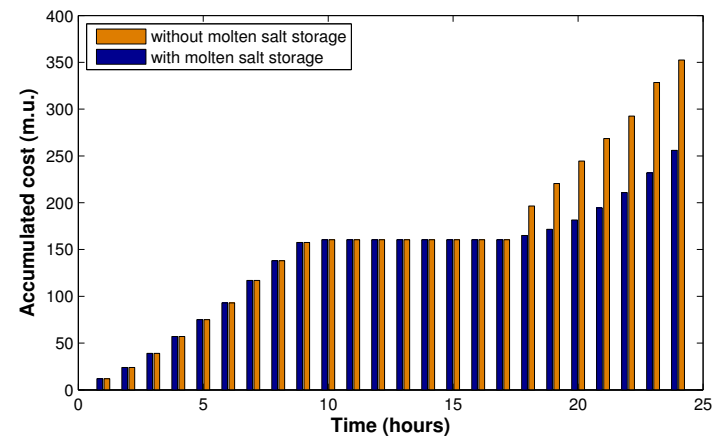

Fig. 12. Interconnections between hubs $\mathrm{i}$ and $\mathrm{j}$

capabilities and without it. As can be seen, accumulated costs are notably higher without considering storage. Such a cost increment is due to the fact that the excess of sun power collected during the central daily time is lost when no storage capabilities are considered. The resulting energy mix corresponding to the last part of the day would be then mainly composed of costly fuel oil power, which is the reason of the theoretical accumulated energy cost increment.

\section{CONCLUSION}

This paper deals with the optimal integration of solar power plants in grids. The paper proposed a modification of energy hubs which allows to solve the optimization problem encountered when integrating solar power plants in electrical grids with a mixed integer programming algorithm in a distributed way. An introductory simulation study case was presented.

\section{ACKNOWLEDGEMENTS}

The author would like to acknowledge the Spanish Ministry of Education, Junta de Andalucia and the European Commission for funding this project under grants DPI2008-05818, P07-TEP-02720 and HD-MPC.

\section{REFERENCES}

S. Massoud Amin and B. F. Wollenberg. Toward a smart grid: power delivery for the 21st century. IEEE Power Energy Mag, 3(5):34-41, 2005.

E.F. Camacho and C. Bordons. Model Predictive Control. Springer Verlag, London, 2004.

E.F. Camacho, M. Berenguel, and F.R. Rubio. Advanced Control of Solar Power Plants. Springer Verlag, London, 1997.

T. F. Garrity. Innovation and trends for future electric power systems. In Proc. PSC'09. Power Systems Conference, pages 1-8, 2009.

M. Houwing, A. N. Ajah, P. M. Herder, and I. Bouwmans. Addressing uncertainties in the design and operation of residential distributed energy resources: Case study of a micro-CHP system. In 10th Conference on Process Integration, Modelling and Optimisation for Energy Saving and Pollution Reduction, Ischia Island, Italy, June 2007.

R.R. Negenborn. Multi-Agent Model Predictive Control with Applications to Power Networks. PhD thesis, Technische Universiteit Delft, 2007. 